\title{
Scheduling and Precoding in Hybrid Analog-Digital Multiantenna Spectrum Sharing Systems
}

\author{
Miguel Ángel Vázquez ${ }^{\star}$, Ana Pérez-Neira ${ }^{\star \dagger}$, Roberto Corvaja ${ }^{\dagger \dagger}$, Ana Garcia Armada ${ }^{\star \star}$, Miguel Ángel Lagunas ${ }^{\star \dagger}$ \\ ${ }^{\star}$ Centre Tecnològic de Telecomunicacions de Catalunya (CTTC/CERCA) \\ $\dagger$ Dept. of Signal Theory and Communications Universitat Politècnica de Catalunya

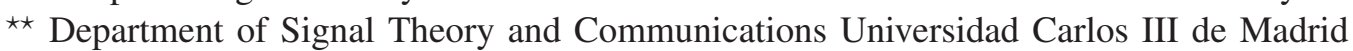 \\ $\dagger^{\dagger}$ Department of Information Engineering University of Padova \\ Email:\{mavazquez\}ecttc.cat
}

\begin{abstract}
This paper investigates scheduling and precoding techniques via hybrid analog-digital transmitters in mm-wave frequency bands. As in spectrum sharing sub-6GHz scenarios, the presence of non-intended receivers limits the overall achievable rates. In order to circumvent this problem, we propose a scheduling and precoding algorithm able to maximize the sumrate while keeping the interference to the external users under a certain threshold. The method consists of a first scheduling algorithm followed by the optimization of the analog and digital beamforming parts. Numerical simulations validate the conceived technique and they show that data rates are increased compared to current designs.
\end{abstract}

\section{INTRODUCTION}

The shared use of the mm-wave spectrum is currently being investigated by both academia and industry. Despite its enormous available bandwidth, spectrally efficient deployments are targeted considering the future exponential user data rate demands. This is the case of hybrid satellite-terrestrial backhaul wireless services where the satellite user terminals and fixed wireless links could eventually share a large portion of the 18 and $28 \mathrm{GHz}$ band spectrum [1].

As for the sub-6 GHz scenarios, interference mitigation techniques are mandatory for enabling the shared use of the spectrum in mm-wave bands [2]. Note that, the mm-wave transmission scenario entails an additional challenge design: the use of hybrid analog-digital precoding schemes which reduce the number of baseband processing inputs in order to alleviate the computational resources and equipment cost.

In order to enhance the user data rates over the conceived interference mitigation techniques, the system designer could opt to perform scheduling techniques based on the intended and non-intended channel vectors. Indeed, the seminal work in [3] show that in a spectrum sharing multiuser multiantenna scenario, scheduling can substantially increase the achievable data rates.

The aim of this paper is to revisit the scheduling spectrum sharing algorithms considering the mm-wave scenario. Precisely, we re-conceive the overall transmission scheme considering the hybrid analog-digital precoding structure. As described in the following sections, the analog and digital parts are optimized separately: while the digital part processes a zero forcing precoding technique, the analog beamforming network steers its beam towards the intended users keeping the interference to the non-intended ones under a certain threshold.
Our proposal results novel compared to the current approaches [4]-[7]. None of the mentioned works considered the interference mitigation over non-intended receivers. The proposed technique is based on the preliminary authors works in [2] and it is extended to the multiuser case via an alternative optimization approach. Furthermore, the paper evaluates the scheduling and power control algorithm considering this underlying hybrid analog-digital precoding technique. Numerical evaluations show that a substantial throughput gain can be obtained with the proposed technique compared to a mere joint precoding and scheduling algorithm without mitigating the interference to the non-intended receivers via the analog beamforming network.

The rest of the paper is organized as follows. Section II describes the system model. Section III presents the scheduling, precoding and power control proposed designs. Section IV depicts the numerical evaluations. Section V concludes the paper.

Notation: Throughout this paper, the following notations are adopted. Boldface upper-case letters denote matrices and boldface lower-case letters refer to column vectors. (.. $)^{H}$, $(.)^{T},(.)^{*}$ and $(.)^{+}$denote a Hermitian transpose, transpose, conjugate and diagonal (with positive diagonal elements ) matrix, respectively. $\mathbf{I}_{N}$ builds $N \times N$ identity matrix and $\mathbf{0}_{K \times N}$ refers to an all-zero matrix of size $K \times N$. If $\mathbf{X}$ is a $N \times N$ matrix. $[\mathbf{X}]_{i j}$ represents the $(i$-th, $j$-th) element of matrix $\mathbf{X} . \otimes$, $\circ$ and $\|$.$\| refer to the Kronecker product,$ the Hadamard product and the Frobenius norm, respectively. Vector $\mathbf{1}_{N}$ is a column vector with dimension $N$ whose entries are equal to 1 . vec $(\cdot)$ denotes the vectorization operator.

\section{System Model And Problem Statement}

Let us consider a base station equipped with $Q$ antennas transmitting to a set of $\mathcal{K}$ users. The received signal by the $k$-th user can be modelled as

$$
y_{k}=\sum_{j \in \mathcal{K}} \sqrt{q_{j}} \mathbf{h}_{k}^{H} \mathbf{v}_{j} s_{j}+n_{k},
$$

where $\mathbf{h}_{k} \in \mathbb{C}^{Q \times 1}$ is the channel vector between the base station and the $k$-th receiver, vector $\mathbf{v}_{k} \in \mathbb{C}^{Q \times 1}$ denotes the beamforming that supports the transmission of the symbol sent to the $k$-th which is denoted by $s_{k}$ and assumed to be zero mean and unit norm. Finally, $n_{k}$ is the additive white Gaussian noise with zero mean and variance equal to 1 for all receivers. 
The transmit power of the addressed to $j$-th user is denoted by $q_{j}$.

We consider a wireless backhaul channel model scenario reported in [8] as 'above the roof top' case. The channel vector in a backhaul scenario can be rewritten as

$$
\mathbf{h}=\sum_{n=1}^{S+1} \alpha_{n} \mathbf{a}\left(\theta_{n}, \phi_{n}\right)
$$

where we have dropped the subindex ${ }_{k}$ for ease of notation and $S$ is the total number of scatterers, $\alpha_{n}$ is the complex gain of the $n$-th scatterer, a $\in \mathbb{C}^{Q \times 1}$ is the steering vector of the transmit antenna array. The steering vector depends on the angles of departure (AoD), $\theta_{n}, \phi_{n}$.

For $n=1$ it is considered that the channel is deterministic and it can be obtained via a geometrical reasoning. More precisely, it is assumed that $\alpha_{1}$ is equal to the path-loss and $\theta_{1}, \phi_{1}$, can be computed by knowing the relative position of the transmitter and the receiver.

For $n>1$, the channel offers a random behaviour based on the first ray $(n=1)$. This is, in [8] it is described that the amplitude values can be modelled as

$$
\alpha_{n}=A_{n} e^{\psi_{n} j}
$$

where $A_{n}$ is Rayleigh distributed with mean $\alpha_{1} / 10$ and $\psi_{n}$ is uniformly distributed from 0 to $2 \pi$. Moreover, it is assumed that the number of scatters is fixed and it is $S=4$. Finally, the AoDs for the different scatters $n>1$ are perturbed by an additive Gaussian random variable of zero mean and 5 degrees of standard deviation.

The steering vector a depends on the antenna array structure and the element spacing. Mm-wave links generally operate with planar arrays due to their high directivity. The simplest planar array representation is an uniform rectangular array (URA). These arrays are usually represented in matrix form but it is more convenient to consider their vector formulation as follows

$$
\mathbf{a}_{\mathrm{URA}}(\theta, \phi)=\operatorname{vec}\left(\mathbf{u}(\theta, \phi) \mathbf{v}(\theta, \phi)^{T}\right),
$$

where $\mathbf{u}(\theta, \phi)$ and $\mathbf{v}(\theta, \phi)$ are described in (5) and (6) in the following page.

Parameters $d_{x}$ and $d_{y}$ are the antenna distances in the $x$ and $y$ axis and $N_{x}$ and $N_{y}$ are the number of elements in the $x$ and $y$ axis respectively.

The communication takes place in presence of $L$ nonintended receivers such as satellite user terminals. The channel vector from the base station to the $l$-th non-intended receiver is denoted by $\mathbf{g}_{l}$. In order to foster the spectrum sharing between the satellite and the terrestrial backhaul system, the base station shall restrict the transmit power towards the satellite user terminals so that

$$
\sum_{k \in \mathcal{K}} q_{k}\left\|\mathbf{g}_{l}^{H} \mathbf{v}_{k}\right\|^{2} \leq I_{l}
$$

for $l=1, \ldots, L$.

In contrast to all-digital designs where $\left\{\mathbf{v}_{k}\right\}_{k \in \mathcal{K}}$ are designed to fulfil a sum-power constraint, in here we consider that each beamformer consists of an analog processing part $\mathbf{P} \in \mathbb{C}^{Q \times N_{R F}}$, where $N_{R F}$ is the number of RF chains, and a digital processing part $\mathbf{w}_{k} \in \mathbb{C}^{N_{R F} \times 1}$ where

$$
\mathbf{v}_{k}=\mathbf{P w}_{k} \quad k \in \mathcal{K} .
$$

This analog processing consists of a network of phase shifters which can modify the phase of the baseband signal while maintaining unaltered its amplitude. We model this analog processing with matrix $\mathbf{P}$ whose entries amplitude are either 1 or 0 depending on the network design as we discuss in the following.

Bearing the above description in mind, the aim of this paper is to solve the following optimization problem

$$
\underset{\mathbf{P},\{q\}_{k \in \mathcal{K}},\{\mathbf{w}\}_{k \in \mathcal{K}}, \mathcal{K}}{\operatorname{maximize}} \sum_{k \in \mathcal{K}} \log _{2}\left(1+\mathrm{SINR}_{k}\right)
$$

subject to

$$
\begin{aligned}
& \mathcal{K} \subseteq \mathcal{M} \quad|\mathcal{K}| \leq N_{R F}, \\
& \sum_{k \in \mathcal{K}} q_{k}\left\|\mathbf{g}_{l}^{H} \mathbf{P} \mathbf{w}_{k}\right\|^{2} \leq I_{l}, \quad l=1, \ldots, L, \\
& \sum_{k \in \mathcal{K}} q_{k}\left\|\mathbf{P} \mathbf{w}_{k}\right\|^{2} \leq P_{\max }, \\
& \mathbf{P} \in \mathcal{P},
\end{aligned}
$$

where,

$$
\operatorname{SINR}_{k}=\frac{q_{k}\left|\mathbf{h}_{k}^{H} \mathbf{P} \mathbf{w}_{k}\right|^{2}}{\sum_{j \in \mathcal{K}, j \neq k} q_{j}\left|\mathbf{h}_{k}^{H} \mathbf{P} \mathbf{w}_{j}\right|^{2}+1},
$$

and $\mathcal{M}$ denotes the set users to be served, $P_{\max }$ the maximum available power and $\mathcal{P}$ is the analog beamforming network feasible set. In this paper we consider the following feasible sets

$$
\begin{gathered}
\mathcal{P}_{\text {full }}:\left|[\mathbf{P}]_{m, n}\right|^{2}=1, \\
\mathcal{P}_{\text {interleaved }}:\left|[\mathbf{P}]_{m, n}\right|^{2}=\left[\mathbf{1}_{\kappa} \otimes \mathbf{I}_{N_{R F}}\right]_{m, n}, \\
\mathcal{P}_{\text {localized }}:|[\mathbf{P}]|^{2}=\left[\mathbf{I}_{N_{R F}} \otimes \mathbf{1}_{\kappa}\right]_{m, n},
\end{gathered}
$$

for $m=1, \ldots, Q \quad n=1, \ldots, N_{\mathrm{RF}}$ and

$$
\kappa=\frac{Q}{N_{R F}},
$$

which is assumed to be an integer value. In (11), (12) and (13) it is described the connectivity of a fully-connected, an interleaved and a localized beamforming network respectively. Each of these mentioned options presents different losses and performance gains. Due to space limitations, we do not describe them in here. The reader can refer to [9] for an extensive discussion of the different beamforming solutions.

The optimization problem (9) shows a combinatorial computational complexity due to the optimization of $\mathcal{K}$ over $\mathcal{M}$. In contrast to other multiuser MIMO scheduling techniques, (9) additionally presents the challenge of designing the analog beamforming network, which is known to be a difficult nonconvex problem even for the single user case [2]. For tackling both the scheduling and the precoder design, we consider alternatives that require low computational resources as we detail in the following. 


$$
\begin{aligned}
\mathbf{u}(\theta, \phi) & =\frac{1}{\sqrt{N_{x}}}\left(1, e^{j \frac{2 \pi}{\lambda} d_{x} \sin (\theta) \cos (\phi)}, \ldots, e^{j \frac{2 \pi}{\lambda}\left(N_{x}-1\right) d_{x} \sin (\theta) \cos (\phi)}\right)^{T} \\
\mathbf{v}(\theta, \phi) & =\frac{1}{\sqrt{N_{y}}}\left(1, e^{j \frac{2 \pi}{\lambda} d_{y} \sin (\theta) \sin (\phi)}, \ldots, e^{j \frac{2 \pi}{\lambda}\left(N_{y}-1\right) d_{y} \sin (\theta) \sin (\phi)}\right)^{T}
\end{aligned}
$$

\section{LOW COMPLEXITY HyBRID ANALOG-DigitAL Multiuser PRECODING}

\section{A. Scheduling}

While greedy scheduling approaches sequentially select the users to be served and compute the resulting beamforming and power allocation iteratively, in this paper we consider the indirect approach, where the scheduler performs its task separately of the beamforming and power control design. More precisely, we select the users based on the co-linearity between their channel vectors and the non-intended users channel vectors.

The procedure consists of two parts. First, the scheduler randomly selects one user $i \in \mathcal{M}$. Posteriorly, the scheduler selects additional users so that

$$
\Delta\left(\mathbf{h}_{i}, \mathbf{h}_{j}\right)=\frac{\left|\mathbf{h}_{i}^{H} \mathbf{h}_{j}\right|}{\left\|\mathbf{h}_{i}\right\|\left\|\mathbf{h}_{j}\right\|} \leq \delta_{\mathrm{t}},
$$

for $j \in \mathcal{M}-\{i\}$. The selected users are denoted by $\mathcal{S}$. The cardinality of $\mathcal{S}$ depends on $\delta_{\mathrm{t}}$ and in general $|\mathcal{S}|>0$.

If $|\mathcal{S}| \leq N_{R F}$ the users to be served are the ones in $\mathcal{S}$. There might be cases where the set $\mathcal{S}$ becomes empty. For that case it shall be increased the value of $\delta_{\mathrm{t}}$. On the other hand, in case $|\mathcal{S}|>N_{R F}$, we perform an additional selection process. We propose to select $N_{R F}$ users considering the spatial signature of the non-intended user terminals. Mathematically, we will choose the users $n \in \mathcal{S}$ so that

$$
\Delta\left(\mathbf{g}_{l}, \mathbf{h}_{n}\right) \leq \delta_{\mathrm{s}},
$$

for all $l=1, \ldots, L$. To sum up, among the overall set of users to be served, $\mathcal{M}$, we select $\mathcal{S}$ with the semi-orthogonal scheduling method and, in case $|\mathcal{S}|>N_{R F}$, we re-select the ones with the lowest co-linearity with the non-intended receiver for obtaining $\mathcal{K}$. The scheduling algorithm is summarized in Algorithm 1.

Note that in the first phase, the number of pre-selected users is increased so that the comparison inside the for loop increases its computational complexity every time there is a new selected user $i$. Furthermore, there is no guarantee of any certain cardinality on $\mathcal{K}$. In other words, in case Algorithm 1 yields to $\mathcal{K}=\emptyset$, it is necessary to increase $\delta_{\mathrm{t}}$ and $\delta_{\mathrm{s}}$ for electing some users.

\section{B. Beamforming}

Once the user selection is performed as the previous section describes, it is time to compute both the analog and digital beamforming weights. These precoding matrices (i. e. $\mathbf{P}, \mathbf{W}$ ) shall be computed based on $\left\{\mathbf{h}_{k}\right\}_{k \in \mathcal{K}}$, which can be collapsed in the following matrix $\mathbf{H}=\left(\mathbf{h}_{1}^{T}, \ldots, \mathbf{h}_{|\mathcal{K}|}^{T}\right)$.

The beamforming network $\mathbf{P}$ in multiuser scenarios has been designed to steer its vectors into different users [4], [5]

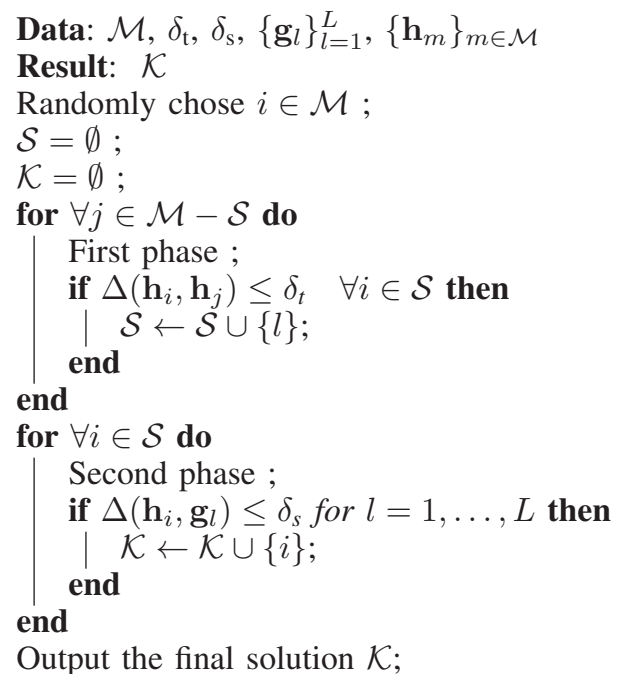

Algorithm 1: Scheduling for spectrum sharing mm-wave systems.

under a fully-connected solution. In this paper, we consider an arbitrary connectivity matrix so that the analog processing formed by the phase-only weights becomes

$$
[\mathbf{P}]_{m, n}=[\mathbf{C}]_{m, n} e^{j \angle\left\{\left[\mathbf{H}^{H}\right]_{m, n}\right\}}
$$

for $m=1, \ldots, Q n=1, \ldots, K$ and where $\angle\{a\}$ denotes the angle of the complex number $a$ and $\mathbf{C}$ collapses the connectivity matrix depending on the beamforming network design described in (11), (12) and (13). It is important to remark that the aim of the analog design in (17) is to steer its beams towards the intended users independently of the underlying connectivity matrix $\mathbf{C}$.

Nevertheless, with the proposed design in (17), there might be the case where the analog beamforming array gain towards non-intended users becomes high, limiting the overall system throughput. As an alternative to the design in (17), we propose to optimize the analog beamforming network so that

$$
\begin{aligned}
& \underset{\mathbf{p}_{k}}{\operatorname{maximize}}\left|\mathbf{h}_{k}^{H} \mathbf{p}_{k}\right|^{2} \\
& \text { subject to } \\
& \left|\left[\mathbf{p}_{k}\right]_{n}\right|^{2}=\left[\mathbf{c}_{k}\right]_{n} \quad n=1 \ldots, N \\
& \left\|\mathbf{g}_{l}^{H} \mathbf{p}_{k}\right\|^{2} \leq I_{l}, \quad l=1, \ldots, L,
\end{aligned}
$$

where $\mathbf{p}_{k}$ and $\mathbf{c}_{k}$ are the $k$-th columns of $\mathbf{P}$ and $\mathbf{C}$ respectively.

The optimization problem in (18) is non-convex due to the equality constraints and the objective function. In order to obtain an approximate solution of (18), we resort to a the penalized convex-concave method (PCC) [10]. This method relies on approximating the non-convex parts of the optimization problem via its convex approximation and incorporating 
slack variables such that

$\underset{\mathbf{p}_{k}, \mathbf{s}}{\operatorname{minimize}}-\operatorname{Re}\left\{\mathbf{z}^{(t), H} \mathbf{R}_{k} \mathbf{p}_{k}\right\}+\varphi \mathbf{1}_{N}^{T} \mathbf{s}$

subject to

$\left\|\mathbf{g}_{l}^{H} \mathbf{p}_{k}\right\|^{2} \leq I_{l}, \quad l=1, \ldots, L$,

$\mathbf{p}_{k}^{H} \mathbf{E}_{m} \mathbf{p}_{k} \leq\left[\mathbf{c}_{k}\right]_{m} \quad m=1, \ldots, Q N_{R F}$,

$\mathbf{p}_{k}^{H} \mathbf{E}_{m} \mathbf{p}_{k} \leq[\mathbf{s}]_{m}+\left[\mathbf{c}_{k}\right]_{m}+2 \operatorname{Re}\left\{\mathbf{z}^{(t), H} \mathbf{E}_{m} \mathbf{p}_{k}\right\} \quad m=1, \ldots, N$,

$[\mathbf{s}]_{m} \geq 0 \quad m=1, \ldots, N$,

where $\mathbf{R}_{k}=\mathbf{h}_{k} \mathbf{h}_{k}^{H}$ and $\mathbf{E}_{m}$ is a $N \times N$ zero matrix whose $m$ th diagonal element is equal to one. As described in [10], PCC method consists of a sequence of second order cone programs that depend on the previous solution $\mathbf{z}$. Due to the impossibility of having an initial feasible value $\mathbf{z}^{(0)}$, slack variables are included into the concave parts of the optimization problem. These slack variables are minimized in the objective function and $\varphi$ controls the balance between the optimization of the objective function and the slack variable minimization.

The overall method is described in Algorithm 2. As in [10] this method is not guaranteed to converge to a feasible solution. In order to always obtain a feasible solution, we include a clause so that the algorithm does not finish till $\mathbf{1}_{N}^{T} \mathbf{s} \leq \chi$. On the other hand, it might be the case where the optimization sequence of Algortihm 2 does not lead to a feasible point. In this case, we consider a maximum number of iterations $T_{\max }$ and, in case they are reached, the algorithm starts again with a new random initial point $\mathbf{z}^{(0)}$.

Data: $\mathbf{z}^{(0)}$ which can be randomly obtained and $\varphi^{(0)}$

Result: p $^{*}$

initialization ;

while $\left|\|\mathbf{p}\|^{(t)}-\|\mathbf{p}\|^{(t-1)}\right| \leq \mu$ and $\mathbf{1}_{N}^{T} \mathbf{s} \leq \chi$ do

if $t<T_{\max }$ then

Compute $\mathbf{p}^{(t)}$ according to (19).;

$\mathbf{z}^{(t+1)} \leftarrow \mathbf{p}^{(t)}$

$\varphi^{(t+1)} \leftarrow \varphi^{(t)} \rho$

$t \leftarrow t+1$

else

$t \leftarrow 0$

Initialize with a new random value $\mathbf{z}^{(0)}$

Set up $\varphi^{(0)}$ again;

end

end

Output the final solution;

Algorithm 2: CCP optimization for analog beamforming.

With these analog processing schemes, the transmitter is able to form the analog beamforming network so that $\mathbf{P}=\left(\mathbf{p}_{1} \ldots, \mathbf{p}_{|\mathcal{K}|}\right)$. In case $|\mathcal{K}|=N_{R F}$ the analog design is finished but, in case $|\mathcal{K}|<N_{R F}$ there are $N_{R F}-|\mathcal{K}|$ analog beamforers to be designed. For this case, we opt for completing the analog rows of $\mathbf{P}$ via copying the consecutive first columns. Mathematically,

$$
\mathbf{P} \leftarrow\left(\mathbf{P}, \mathbf{P}_{\mathrm{a}}\right),
$$

where $\mathbf{P}_{\mathrm{a}}$ is a matrix formed by the first $N_{R F}-|\mathcal{K}|$ columns of $\mathbf{P}$.
Once the analog processing is done, the digital part observes an equivalent channel matrix

$$
\mathbf{H}_{\mathrm{eq}}=\mathbf{H P} \text {. }
$$

For the digital processing part we consider the sub-optimal low complex zero-forcing approach so that

$$
\mathbf{W}_{\mathrm{ZF}}=\rho\left(\mathbf{H}_{\mathrm{eq}}^{H} \mathbf{H}_{\mathrm{eq}}\right)^{-1} \mathbf{H}_{\mathrm{eq}}^{H} .
$$

Finally, when the scheduling and precoding operations are done, the power allocation is performed via the following optimization problem

$$
\begin{aligned}
& \underset{\mathbf{q}}{\operatorname{maximize}} \sum_{k \in \mathcal{K}} \log _{2}\left(1+\mathrm{SINR}_{k}\right) \\
& \text { subject to } \\
& \sum_{k \in \mathcal{K}} q_{k}\left\|\mathbf{g}_{l}^{H} \mathbf{P} \mathbf{v}_{k}\right\|^{2} \leq I_{l}, \quad l=1, \ldots, L, \\
& \sum_{k \in \mathcal{K}} q_{k}\left\|\mathbf{P} \mathbf{w}_{k}\right\|^{2} \leq P_{\max },
\end{aligned}
$$

which is known to be solvable via water-filling methods. Due to space limitations, we do not describe the solution of (23) in here.

\section{NUMERICAL RESULTS}

In this section we evaluate numerically the proposed transmission mechanisms. We consider a transmitter equipped with $N=64$ antennas with $N_{R F}=4$ in presence of $|\mathcal{M}|=100$ possible receivers and $L=1$ non-intended receivers. The simulation results have been obtained with an average of 500 Monte Carlo runs and a variable transmit power of $P_{\max }=2, \ldots, 16 \mathrm{dBWatts}$. Both the intended and nonintended user channel vectors are obtained with the described channel modelling in Section II.

We first evaluate the precoding performance design comparing the design in (17) coined as Benchmark in the figures and the proposed based on the CCP optimization in Algorithm 2. For this comparison, we consider the figure of merit of the sum-rate computed as

$$
\mathcal{S R}=\sum_{i \in \mathcal{S}} \log _{2}\left(1+\operatorname{SINR}_{i}\right)
$$

The parameters used in Algorithm 2 are $\varphi^{(0)}=10, \rho=2$, $\mu=10^{-3}, \chi=10^{-2}, T_{\max }=30$. These values have been obtained through different simulation trials and they are the ones that offer the best simulation time versus performance trade-off. As an initial point, we consider

$$
\mathbf{z}^{(0)}=\operatorname{vec}\left(\mathbf{P}_{\text {benchmark }}\right),
$$

obtained as described in (17). In addition, we consider that $I_{1}=10^{-2}$.

The precoding evaluation with no scheduling is described in Figure 1. As it can be observed, the proposed analog beamforming design based on PCC shows a superior performance compared to the benchmark heuristic approach. Among the different analog beamforming networks, the fully-connected alternative presents larger spectral efficiency compared to 


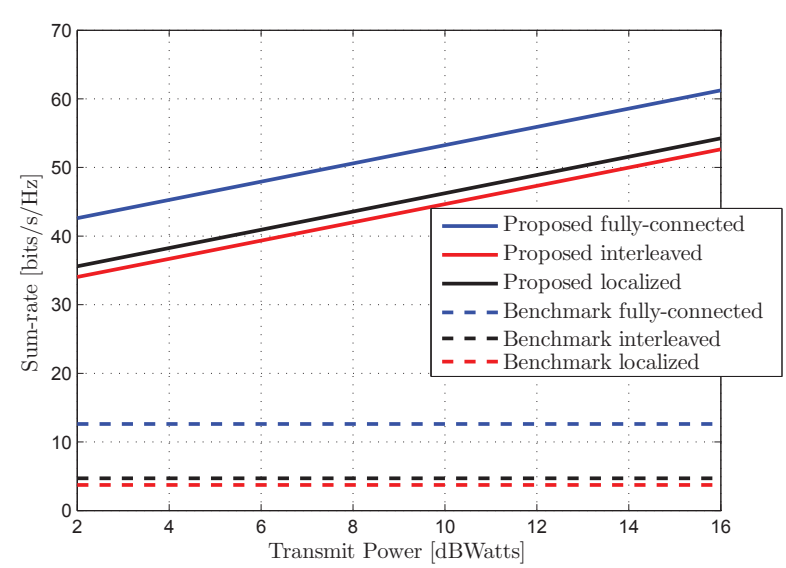

Fig. 1. Sum-rate versus transmit power for different analog beamforming solutions. It is considered $L=1, Q=64$ and $N_{R F}=4$. No scheduling algorithm is considered in this simulation. Different analog beamforming options are shown

the partially-connected ones both in the benchmark and the proposed solution.

Bearing in mind that the fully-connected alternative with the proposed analog optimization is the one that offers the largest sum-rate, we next evaluate the performance of the scheduling technique. In Figure 2 it can be observed the sumrate when no scheduling is performed and when the proposed scheduling method is used with $\delta_{t}=\delta_{s}$ taking different values. Remarkably, for the lowest value $\delta_{t}=\delta_{s}=0.05$ the scheduling technique yields a lower sum-rate compared to the case where the users are randomly selected. On the other hand, for higher values (i.e. 0.06 and 0.07) a sum-rate gain can be appreciated over all transmit power. Precisely a $6 \%$ gain in sum-rate is observable over the transmit power.

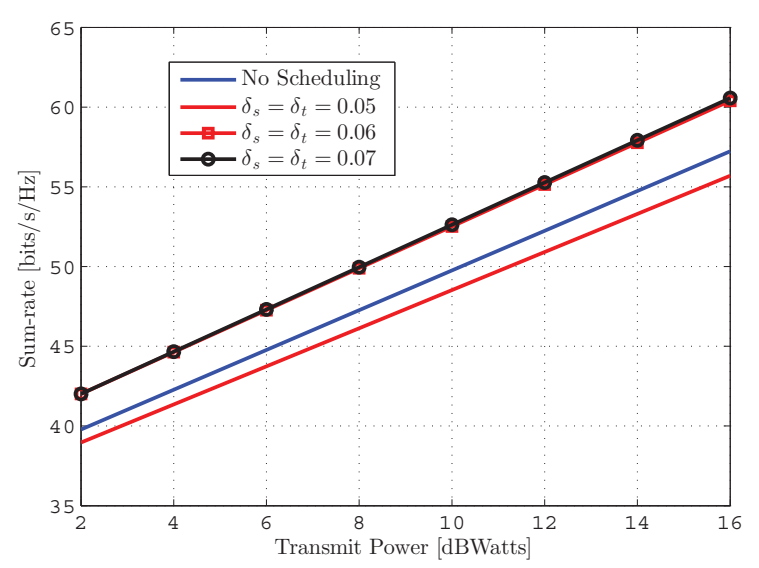

Fig. 2. Sum-rate versus transmit power for different $\delta_{s}$ and $\delta_{t}$. It is considered $L=1, Q=64$ and $N_{R F}=4$. In here it is considered a fullyconnected analog beamforming network and the proposed analog beamforming optimization.

Interestingly, for every scenario with different $L, Q, N_{R F},\left\{I_{l}\right\}_{l=1}^{L}, P_{\max }$ there would be an efficient pair $\delta_{t}, \delta_{s}$ that maximizes the sum-rate. Due to space limitations we only show the above mentioned scenarios and the evaluation in more realistic use cases is left for further works.

\section{CONCLUSIONS}

This paper proposes a transmission mechanism for mmwave spectrum sharing scenarios. In contrast to general wireless transmission alternatives where the processing is done at the base-band, mm-wave transceivers require hybrid analogdigital precoding techniques. Two analog designs are proposed which can deal with an arbitrary connectivity analog beamforming network. Based on the numerical results, the proposed multiuser precoding designs, jointly with the scheduling method, show an enormous advantage in next generation spectrum sharing backhauling networks.

\section{ACKNOWLEDGMENT}

This work has received funding from the European Unions Horizon2020 research and innovation programme under grant agreement No 645047(SANSA); the Spanish Ministry of Economy and Competitiveness (Ministerio de Economia y Competitividad) under project TEC2014-59255-C3-1-R and TEC201459255-C3-3-R (ELISA); and from the Catalan Government (2014SGR1567 and 2014SGR1551)

\section{REFERENCES}

[1] X. Artiga, J. Nunez-Martinez, A. Perez-Neira, G. J. L. Vela, J. M. F. Garcia, and G. Ziaragkas, "Terrestrial-satellite integration in dynamic 5G backhaul networks," in 2016 8th Advanced Satellite Multimedia Systems Conference and the 14th Signal Processing for Space Communications Workshop (ASMS/SPSC), Sept 2016, pp. 1-6.

[2] M. A. Vázquez, L. Blanco, X. Artiga, and A. Pérez-Neira, "Hybrid analog-digital transmit beamforming for spectrum sharing satelliteterrestrial systems," in 2016 IEEE 17th International Workshop on Signal Processing Advances in Wireless Communications (SPAWC), July 2016, pp. 1-5.

[3] K. Hamdi, W. Zhang, and K. B. Letaief, "Opportunistic spectrum sharing in cognitive MIMO wireless networks," IEEE Transactions on Wireless Communications, vol. 8, no. 8, pp. 4098-4109, August 2009.

[4] A. Alkhateeb, G. Leus, and R. W. Heath, "Limited Feedback Hybrid Precoding for Multi-User Millimeter Wave Systems," IEEE Transactions on Wireless Communications, vol. 14, no. 11, pp. 6481-6494, Nov 2015.

[5] L. Liang, W. Xu, and X. Dong, "Low-Complexity Hybrid Precoding in Massive Multiuser MIMO Systems," IEEE Wireless Communications Letters, vol. 3, no. 6, pp. 653-656, Dec 2014.

[6] T. E. Bogale, L. B. Le, A. Haghighat, and L. Vandendorpe, "On the Number of RF Chains and Phase Shifters, and Scheduling Design With Hybrid Analog;Digital Beamforming," IEEE Transactions on Wireless Communications, vol. 15, no. 5, pp. 3311-3326, May 2016.

[7] W. Yuan, S. M. D. Armour, and A. Doufexi, "A novel user selection algorithm for multiuser hybrid precoding in mmWave systems," in 2016 IEEE 27th Annual International Symposium on Personal, Indoor, and Mobile Radio Communications (PIMRC), Sept 2016, pp. 1-6.

[8] A. Maltsev and et al, "D5.1 - Channel Modeling and Characterization," MiWEBA Project (FP7-ICT-608637), Public Deliverable, Jan. 2014.

[9] Vázquez, Miguel Ángel and Artiga, Xavier and Pérez-Neira, Ana, "Low-Cost Hybrid Analog-Digital Beamformer Evaluation in Spectrum Sharing Systems," in 2017 11th European Conference on Antennas and Propagation (EuCAP), March 2017, pp. 1-4.

[10] T. Lipp and S. Boyd, "Variations and extension of the convexconcave procedure," Optimization and Engineering, vol. 17, no. 2, pp. 263-287, 2016. [Online]. Available: http://dx.doi.org/10.1007/ s11081-015-9294-x 